\title{
Congestion Management using Genetic Algorithm in Deregulated Power Environments
}

\author{
S.M.H Nabavi ${ }^{1}$ \\ A. Kazemi ${ }^{1}$ \\ M.A.S. Masoum ${ }^{2}$ \\ ${ }^{1}$ Department of Electrical Engineering, Iran University of Science \& Technology, Tehran, Iran \\ ${ }^{2}$ Department of Electrical and Computer Engineering, Curtin University of Technology, Perth, WA, Australia
}

\begin{abstract}
Congestion cost allocation is an important issue in congestion management. This paper presents a genetic algorithm (GA) to determine the optimal generation levels in a deregulated market. The main issue is congestion in lines, which limits transfer capability of a system with available generation capacity. Nodal pricing method is used to determine locational marginal price (LMP) of each generator at each bus. Simulation results based on the proposed GA and the Power World Simulator software is presented and compared for the IEEE 30-bus test system.
\end{abstract}

Keywords: Congestion management, nodal pricing, deregulated power systems, Genetic algorithm and optimal bidding strategy.

\section{INTRODUCTION}

Transmission congestion will most likely occur when many transactions or scheduled/forced outages exist in the power system. Optimal allocation of congestion cost is usually performed using the branch [1] or the node [2] allocation techniques. The branch allocation method first assigns a system congestion cost (to the congested branch) and then allocates the branch congestion cost (for each transaction) by using sensitivity or tracing methods [3-6]. The node allocation method directly allocates the system congestion cost to the nodes.

Locational market power is a well-known and studied issue in power systems [7-8]. The pricing system (e.g., nodal price or locational marginal price (LMP)) plays an important role in the congestion management [9-12]. Reference [9] proposes a "fixed transmission right" model for the congestion management. Reference [1] presents two approaches based on the pool model and the bilateral model. Reference [10] provides an operation decision support software system covering the functionality of transmission dispatch and congestion management system (TDCMS). In [11], both real and reactive power controllers are considered to mitigate the transmission congestion using optimization. This document also discusses three possible curtailment strategies.

In recent years the methods of managing transmission congestion have been under intense scrutiny [12-21, 25]. Presently there are two distinct congestion management systems widely being employed: nodal pricing [20] and zonal pricing methods [13]. Many of these congestion management models are based on optimal power flow algorithms [13-14]. A primal- dual interior point linear programming method is applied to solve the congestion model in [15]. This method can also be used to solve real-time congestion. The impacts of load demand elasticity on congestion management and pricing are investigated in [16].

Genetic algorithms (GAs) are widely accepted as an effective optimization method to solve various types of large-scale engineering problems. Different congestion management methods based on genetic algorithms are presented in references [17-21]. The problem of building optimal bidding strategies for generation companies has also been formulated as a stochastic optimization model and solved by the well-known Monte Carlo simulation method and Genetic Algorithms [17, 24].

This paper proposes a new genetic algorithm to minimize the total system cost with congestion in a deregulated market. Simulation results of the genetic algorithm (for 30-bus test system) are compared with those provided by the Power World Simulator [22] to demonstrate the applicability and efficiency of the proposed method for congestion management in a deregulated electricity market.

\section{NODAL PRICING}

In the nodal pricing method, system operator sets the electricity price and determines the dispatch level of each generator based on the requested marginal supply bids which is a monotonically increasing curve indicating supplier's individual preference on the production amount at various prices. Consequently, the price and dispatch amount are used to determine an economically optimal operating point while respecting system constraints. Nodal pricing method of managing transmission congestion is based on the computation of location marginal price at each individual node of the power system. The market-clearing price is marginal cost of the marginal unit in the absence of transmission constraints. In economics terms, the marketclearing price is the point of intersection of supply and demand curves. However, in the presence of transmission constraints, prices vary by the location since cost of producing energy differs [20]. Nodal pricing applies spatial spot pricing theory on a real time basis to derive a bus-by-bus LMP [20].

\section{II.1. Locational Marginal Price}

LMP is the marginal cost of supplying the next increment of electric energy at a specific bus, considering the generation marginal cost and the physical aspects of the transmission system. Marginal pricing reflects the cost to serve the next increment of load in a system that is economically dispatched. Three factors influencing LMP are "marginal cost to operate 
generation", "total load" and "cost of delivery". LMP is defined as [20]:

$\mathrm{LMP}=$ generation marginal cost

+ congestion cost + cost of marginal losses

LMP is the dual variable for the equality constraint at a node (e.g., sum of injections and withdrawals is equal to zero). Both loss and congestion components are always zero at the reference bus. Therefore, the price at the reference bus is always equal to the energy component. LMPs will not change if the reference bus is allocated. However, all three components of LMP dependent on the selection of the reference bus due to the dependency of sensitivities on the location of reference bus. In fact, LMP is the additional cost for providing additional MW at a certain bus [22].

Using LMP, buyers and sellers experience the actual price of delivering energy to locations on the transmission systems. If the line flow constraints are not included in the optimization problem, LMPs will be the same for all buses. This is the marginal cost of the most expensive dispatched generation unit (marginal unit). In this case, no congestion charges apply. However, if any line is constrained, LMPs will vary from bus to bus and may cause congestion charges [21].

\section{II.2. LMP Price Calculation Procedures}

At any bus i, $L M P_{i}$ is composed of three components; marginal generation price at the reference bus ( $\left.L M P_{i}^{\text {ref }}\right)$, loss component $\left(L M P_{i}^{\text {loss }}\right)$, and congestion component $\left(L M P_{i}^{\text {cong }}\right)$ :

$L M P_{i}=L M P_{i}^{\mathrm{ref}}+L M P_{i}^{\text {loss }}+L M P_{i}^{\text {cong }}$

where values of the three components are based on the selection of reference bus. The last two components are given as:

$$
\begin{aligned}
& L M P_{i}^{\text {loss }}=\left(D F_{i}-1\right) L M P_{i}^{\text {ref }} \\
& L M P_{i}^{\text {cong }}=-\sum_{k \in K} G S F_{i k} \beta_{k}
\end{aligned}
$$

Where

- $D F_{i}$ is the delivery factor of bus i relative to reference bus (e.g., a measure of the portion of the next MW generation at buses $i$ that is delivered to the reference bus).

- $G S F_{i k}$ is the generation shift factor for bus i on line k (e.g., ratio of the change in flow of line $\mathrm{k}$ to the change in generation of bus i). All generation shift factors at the reference bus are equal to zero [21].

- $k$ is the set of congested transmission lines.

- $\beta_{k}$ is the constraint cost of line $\mathrm{k}$, defined as $\beta=\frac{\text { Reduction in total } \cos t}{\text { Change in constriant's flow }}$.

\section{PROBLEM FORMULATION}

\section{III.1 Objective Function}

LMP is determined by solving the following optimization problem:

$$
\min _{Q_{G_{i}}} \sum_{G_{i}} C_{G_{i}}\left(P_{G_{i}}\right) \quad \text { fo } \dot{\pi}=1, \ldots, n
$$

where $P_{G_{i}}$ is the amount of dispatched generation at node $G_{i}$ and $C_{G_{i}}$ is the total cost of generation at node $G_{i}$ (expressed in terms of $P_{G_{i}}$ ).

\section{III.2 Constraints}

The optimization problem is subjected to a number of constrains that are discussed next.

Load flow constraint (total generation=system load):

$\sum_{G_{i}} P_{G_{i}}=\sum_{D_{i}} P_{D_{i}} \quad$ for $i=1, \ldots, n$

Transmission line flow constraints (e.g., power flow on line $l$ is within the maximum line rating):

$$
\left|F_{l}\right|=\left|H_{l_{G_{i}}} P_{G_{i}}+H_{l_{D_{i}}} P_{D_{i}}\right| \leq F_{l}^{\max } \text { for } i=1, \ldots, n
$$

Generation limit constraints (e.g., dispatch amount at node $G_{i}$ is within the maximum rating of the corresponding generator):

$$
P_{G_{i}}^{\text {min }} \leq P_{G_{i}} \leq P_{G_{i}}^{\max } \quad \text { for } i=1, \ldots, n
$$

For simplicity, DC power flow is used for computing the flows on each line of the system. The DC power flow equations in matrix notation are written as:

$$
B \delta=P_{G_{i}}-P_{D_{i}}
$$

where $\delta$ is the voltage angle vector, $P_{G_{i}}$ is the real power generation vector for buses $G_{i}$ and $P_{D_{i}}$ is the real power load vector for buses $D_{i}$

Therefore, flow vectors for lines can be computed as:

$F_{1}=H \delta$

where $H$ is the linearized flow matrix for the system.

If the generation cost of supplier $G_{i}$ (e.g., $C_{G_{i}}$ ) is a function of the output given by:

$$
C_{G_{i}}\left(P_{G_{i}}\right)=a+b P_{G_{i}}+c P_{G_{i}}^{2}+d P_{G_{i}}^{3}
$$

Then, assuming perfect competitive market conditions, the optimal supply bid by supplier $G_{i}$ (e.g., $M C_{i}$ ) is the marginal cost bid given by:

$$
M C_{i}=b+c P_{G_{i}}+d P_{G_{i}}^{2}
$$




\section{PROPOSED GENETIC ALGORITHM}

Genetic algorithms use the principle of natural evolution and population genetics to search and arrive at a high quality near global solution. The required design variables are encoded into a binary string as a set of genes corresponding to chromosomes in biological systems. Unlike the traditional optimization techniques that require one starting point, they use a set of points as the initial conditions. Each point is called a chromosome. A group of chromosomes are called a population. The number of chromosomes in a population is usually selected to be between 30 to 300 . Each chromosome is a string of binary codes (genes) and may contain substrings. The merit of a string is judged by the fitness function, which is derived from the objective function and is used in successive genetic operations. During each iterative procedure (referred to as generation), a new set of strings with improved performance is generated using three GA operators (namely reproduction, crossover and mutation).

\section{IV.1 Structure of Chromosomes}

In this paper, the chromosome structure for GA consists of MC substrings of binary numbers (Fig. 1), where MC denotes amount of generation at each generator bus.

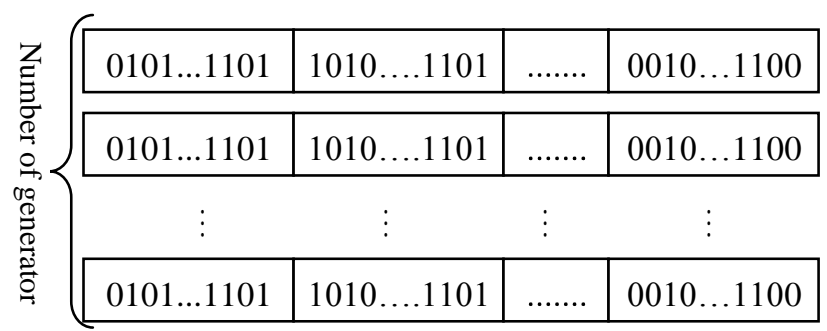

Figure 1: Proposed chromosome structure for the genetic algorithm

\section{IV.2 Proposed Fitness Function}

In this paper, exponential penalty functions are used as the fitness function to combine the objective and constraints:

$$
\begin{aligned}
& F_{\text {fitness }}=\frac{1}{F_{L} \cdot F_{G}} \\
& F_{L}=\prod_{j=1}^{b} F_{\text {line flow }} \\
& F_{G}=\prod_{j=1}^{m} F_{\text {generation limit }}
\end{aligned}
$$

where $\mathrm{b}$ and $\mathrm{m}$ are the number of branches and generators in the power system, respectively.

\section{IV.3 Genetic Operators}

Genetic operators are the stochastic transition rules applied to each chromosome during each generation procedure to generate a new improved population from an old one. A genetic algorithm usually consists of reproduction, crossover and mutation operators.

- Reproduction- is a probabilistic process for selecting two parent strings from the population of strings on the basis of "roulette-wheel" mechanism, using their fitness values. This ensures that the expected number of times a string is selected is proportional to its fitness relative to the rest of the population. Therefore, strings with higher fitness values have a higher probability of contributing offspring.

- Crossover- is the process of selecting a random position in the string and swapping the characters either left or right of this point with another similarly partitioned string. This random position is called the crossover point. In this paper the characters to the right of a crossover point are swapped. The probabilities of parent-chromosomes crossover are assumed to be between 0.01 and 0.3 .

- Mutation- is the process of random modification of a string position by changing " 0 " to " 1 " or vice versa, with a small probability. It prevents complete loss of genetic material through reproduction and crossover by ensuring that the probability of searching any region in the problem space is never zero. In this paper the probability of mutation is assumed to be between 0.01 and 0.1 .

\section{IV.4 Convergence Criterion}

The iterations (regenerations) of the proposed genetic algorithm are continued until all generated chromosomes become equal or the maximum number of iterations is achieved $\left(N^{\max }=40\right)$. Due to the randomness of GA method, the solution tends to differ for each run, even with the same initial population. For this reason, it is suggested to perform multiple runs and select the "most acceptable" solution (e.g., with most benefits, within the permissible region of constraints).

\section{SOLUTION METHODOLOGY}

The cost minimization problem is solved using the proposed genetic algorithm, as follows:

Step 1: input system parameters (e.g., system topology, line and load specifications, maximum and minimum generation limits, and line flow limits). Input the initial population with $N_{\text {chrom }}$ chromosomes.

Step 2: Set initial counter and parameter values (e.g., $\mathrm{N}_{\mathrm{ch}}=\mathrm{N}_{\mathrm{it}}=\mathrm{N}_{\mathrm{R}}=1$ and $F_{M I N}=$ a high number)

Step 3 (Fitness Process):

Step 3A: Run DC power flow for chromosome $N_{c h}$ and save outputs.

Step 3B: Compute proposed penalty functions (Fig. 2) using outputs of the DC power flow. Compute fitness functions (Eq. 12) for chromosome $\mathrm{N}_{\mathrm{ch}}$. Set $N_{c h}=N_{c h}+1$.Step 3C: If $\mathrm{N}_{\mathrm{ch}} \leq \mathrm{N}_{\text {chrom }}$ go to Step 3A.

Step 4 (Reproduction Process):

Step 4A: Define total fitness as the sum of all fitness values for all chromosomes.

Step 4B: Select a percentage of "roulette wheels" for each chromosome, which is equal to the ratio of its fitness value to the total fitness value. 
Step 4C: Improve generation by rolling the "roulette wheel" $N_{\text {chrom }}$ times. Select a new combination of chromosomes.

Step 5 (Crossover Process):

Step 5A: Select a random number $\left(R N D_{1}\right)$ for mating two parent chromosomes.

Step 5B: If $R N D_{1}$ is between 0.01 and 0.3 then combine the two parents, generate two offspring and go to Step 5D.

Step 5C: Else, transfer the chromosome with no crossover.

Step 5D: Repeat steps 5A to 5C for all chromosomes.

Step 6 (Mutation Process):

Step 6A: Select a random number $\left(R N D_{2}\right)$ for mutation of one chromosome.

Step 6B: If $R N D_{2}$ is between 0.01 and 0.1 then apply the mutation process at a random position and go to Step 6D.

Step 6C: Else, transfer the chromosome with no mutation. Step 6D: Repeat Steps 6A to 6C for all chromosomes.

Step 7 (Updating Populations): Replace the old population with the improved population generated by Steps 2 to 6 . Check all chromosomes, if there is any chromosome with $F_{L}=1, F_{G}=1$ and $F_{F}<F_{M I N}$, set $F_{M I N}=F_{F}$ and save it. Set $N_{i t}=N_{i t}+1$.

Step 8 (Convergence): If all chromosomes are the same or the maximum number of iterations is achieved $\left(N_{i t}=N^{\mathrm{max}}\right)$, then print the solution and stop, else go to Step 2.

\section{SIMULATION RESULTS of the 30-bus System}

The proposed methodology has been implemented on IEEE 30 bus system which is shown in Fig. 2 [20] to demonstrate the robustness of proposed method. The network and load data for this system are taken from [20]. Line limits for IEEE 30-bus system are taken from [20]. The results obtained have been found satisfactory.

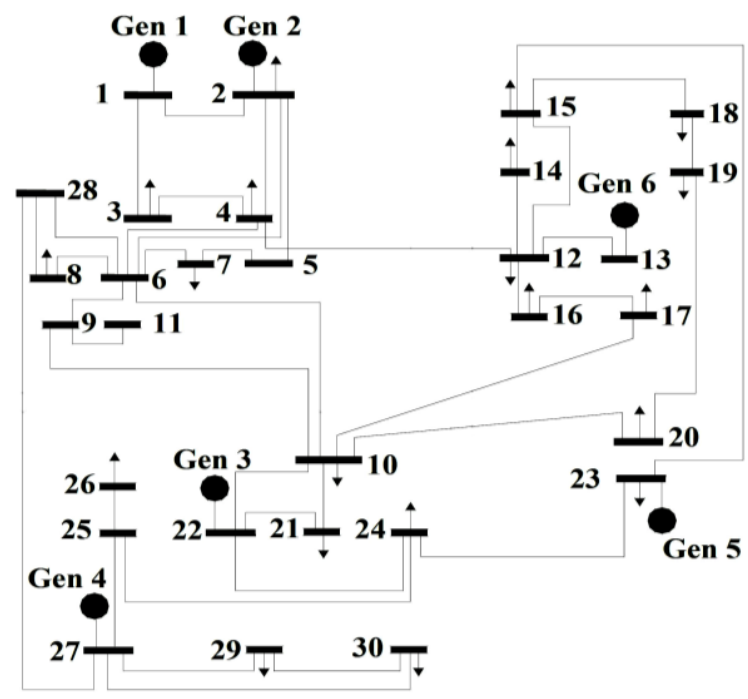

Fig. 2. IEEE 30-bus system [20]

In this system maximum total active load is $232.3 \mathrm{MW}$ and maximum total generation is $395 \mathrm{MW}$. The used genetic parameters for optimization are presented in Table 1 . Table 2 shows simulation results (the system total cost) for the 30-bus system. The Power World Simulator [22] is used to demonstrate that the proposed method is efficient for congestion management in a deregulated electricity market. In addition, Fig. 3 presents simulation of the optimal Generation with minimum cost in congestion mode.

Table 1. GA PARAMETERS

\begin{tabular}{|c|c|}
\hline Population size & 100 \\
\hline Crossover rate & 0.85 \\
\hline Mutation rate & 0.05 \\
\hline Maximum iteration & 500 \\
\hline
\end{tabular}

Table 2: Generation Levels of the 30-bus system after applying the proposed genetic algorithm

\begin{tabular}{|c|c|c|}
\hline $\begin{array}{l}\text { Gen } \\
\text { No. }\end{array}$ & $\begin{array}{c}\text { Generation schedule } \\
\text { (MW) }\end{array}$ & $\begin{array}{c}\text { Total Generation cost } \\
(\$ / \mathbf{h})\end{array}$ \\
\hline G1 & 53.60 & \multirow{7}{*}{1271.16} \\
\hline G2 & 71.00 & \\
\hline G3 & 25.90 & \\
\hline G4 & 26.60 & \\
\hline G5 & 18.00 & \\
\hline G6 & 42.00 & \\
\hline Total & 237.07 & \\
\hline
\end{tabular}

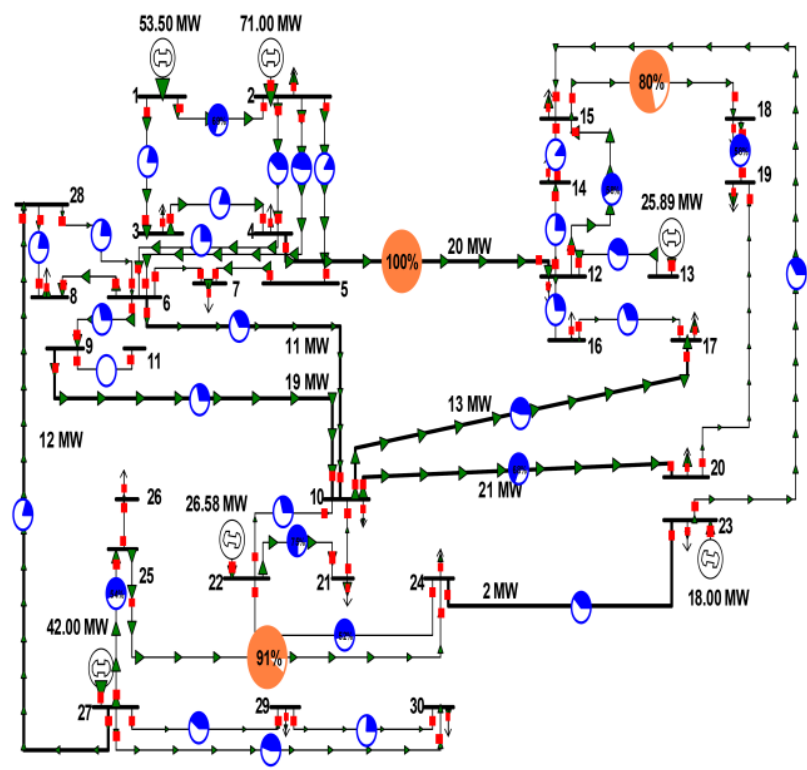

Figure 3: Optimal generation of the 30-bus system with minimum cost 


\section{CONCLUSIONS}

In this paper, a genetic algorithm is proposed and implemented to minimize the total cost of generation in power systems. Nodal pricing method is used to determine marginal cost at each bus. The locational marginal cost of each bus is determined by adding marginal cost of reference bus and the cost due to congestion. Simulation results of the proposed GA for 30-bus IEEE test system indicate a significant reduction in system's total cost to be provided by the consumers (Tables 2 and 3 ). Also, using the Power World Simulator software to illustrate the fine accuracy of the proposed approach for congestion management in deregulated environments.

\section{REFERENCES}

[1] H. Singh, S. Hao, and A. Papalexopoulos, "Transmission congestion management in competitive electricity markets", IEEE Trans. on Power Systems, 1998, Vol. 13, No. 2, pp. 672-680

[2] A.G. Bakirtzis, "Aumann-Shapley transmission congestion pricing”, IEEE Power Eng. Rev., 2001, Vol. 21, No.2, pp. $67-69$

[3] N.S. Rau, "Transmission loss and congestion cost allocation - an approach based on responsibility", IEEE Trans. on Power Systems, 2000, Vol. 15, No. 4, pp. 1401-1409

[4] H. Rudnick, R. Palma, and J.E. Fernandez, "Marginal pricing and supplement cost allocation in transmission open accesses", IEEE Trans. on Power Systems, 1995, Vol. 10, No. 2, pp. 1125-1132

[5] D. Shirmohammadi, P.R. Gribik, and E.T.K. Law, 'Evaluation of transmission network capacity use for wheeling transactions', IEEE Trans. Power Systems, 1989, Vol. 4, No. 4, pp. 1405-1413

[6] M.E. Baran, V. Bannsunarayanan, and K.E. Garren, 'A transaction assessment method for allocation of transmission services', IEEE Trans. Power Systems, 1999, Vol. 24, No. 3, pp. 920-928

[7] H. Singh, "Market power mitigation in electricity markets," Game Theory Applications in Electric Power Markets, IEEE PES Tutorial, TP-136-0, Feb. 1999, pp. 70-76

[8] R. Ethier, R. Zimmerman, T. Mount, W. Schulze, and R. Thomas, "A uniform price auction with locational price adjustments for competitive electricity markets," Int. J. Elect. Power Energy System, Vol. 21, No. 2, pp. 103-110, 1999.

[9] M.J. Alomoush and S.M. Shahidehpour, "Fixed Transmission Right for Zonal Congestion Management," IEE Proceedings-Generation. Transmission \& Distribution, Vol. 146, No. 5, Sep. 1999, pp. 471-476.

[10] D. Shirmohammadi, B. Wollenberg, A. vojdani, P. Sandrin, M. Pereira, F. Rahimi, T. Schneider and B. Stott, "Transmission Dispatch and Congestion Management in the Emerging Energy Market Structures," IEEE Trans. on Power Systems, Vol. 13, No. 4, Nov. 1998, pp. 1466-1474.

[11] R.S. Fang and A.K. David, "Transmission Congestion Management in an Electricity Market," IEEE Trans. on Power Systems, Vol. 14, No. 3, Aug. 1999, pp. 877-883.
[12] T. Gedra, "On Transmission Congestion and Pricing", IEEE Transactions on Power Systems, Vol. 14, No 1, pp. 241248, February 1999.

[13] X. Wang and Y.H. Song, "Apply lagrangian relaxation to multi - zone congestion management", IEEE 2001, Con. 2001, pp. 399-404

[14] I.J.P. Arriaga, F.J. Rubio, J.F. Puerta and J.M. Arceluz, "Marginal Pricing of Transmission Services: An Analysis of Cost Recovery", IEEE Transactions on Power Systems, Vol. 10, No. 1, February 1995

[15] X. Wang, Y.H Song and Q. Lu, "Primal-dual Interior Point Linear Programming Optimal Power Flow for Real-time Congestion Management (C)", IEEE Power Engineering Society Winter Meeting, Singapore, 2000, pp. 1643-1649

[16] E. Bompard, E. Carpaneto, G. Chicco and G. Gross, "The role of load demand elasticity in congestion management and pricing", IEEE Power Engineering Society Summer Meeting, Seattle, WA (USA). 2000, pp. 2229 - 2234

[17] M. Li, F. Wen, N. Yixin and F.F Wu, "Optimal bidding strategies for generation companies in electricity markets with transmission capacity constraints taken into account", IEEE Power Engineering Society General Meeting, 2003, Vol. 4, pp. 13-17, July 2003

[18] J.M. Ramirez and G.A. Marin, "Alleviating congestion of an actual power system by genetic algorithms", Power Engineering Society General Meeting, 2004. IEEE, 6-10 June 2004, Vol. 2, pp. 2133-2141

[19] M. Saguan, S. Plumel, P. Dessante, J.M. Glachant and P. Bastard, "Genetic algorithm associated to game theory in congestion management"; Probabilistic Methods Applied to Power Systems, 2004 International Conference on, 12-16 Sept. 2004 pp. 415-420

[20] M. Shahidehpour, H. Yamin and Z. Li, "Market Operations in Electric Power Systems: Forecasting, Scheduling, and Risk Management", John Wiley \& Sons, Inc., 2002, ISBNs: 0-471-44337-9 (Hardback); 0-471-22412-X (Electronic)

[21] A.J. Wood and B.F. Wollenberg, "Power Generation, Operation, and Control”, New York: Wiley, 1996

[22] R. Coutu, "Locational Marginal Pricing"; ISO New England's Wholesale Energy Market (Intermediate) - WEM 201, Nov 28-30, 2006

[23] Power World Corporation, http://www.powerworld.com

[24] Seyed Abbas Taher, Muhammad Karim Amooshahi, "Optimal Load Frequency Control Using PSO Algorithm in Deregulated Power Systems", Vol. 2. n. 6, pp. 708-714

[25] Iman Mohammad Hoseiny Naveh, Behzad Asadi Zadeh, "Application of FACTS Controllers to Damp Detrimental Resonance in Power System: a Case Study of Supplemental Implement of SVC in a Long AC Interconnection”, Vol. 2. n. 6, pp. 693-699. 